\title{
Are milk polyamines preventive agents against food allergy?
}

\author{
Guy Dandrifosse*, O. Peulen, N. El Khefif, P. Deloyer, A. C. Dandrifosse and Ch. Grandfils \\ Department of Biochemistry and Physiology, Institute of Chemistry, University of Liege, Sart Tilman 4000, Liege, Belgium
}

\begin{abstract}
Insufficient polyamine intake could play a role in the induction of sensitization to dietary allergens. This proposal is based essentially on investigations made in sucking rats and in children. In sucking rats it has been established that oral administration of spermine can induce all the modifications occurring in the digestive tract at weaning. In the intestine events occur in two phases. The early event consists of desquamation of the epithelium resulting from an activation of apoptosis. The late event appears to involve an hormonal cascade in which adrenocorticotropic hormone, cytokines, bombesin and corticosterone are included. Observations in human subjects show that: (1) the spermine and spermidine concentrations are generally lower in infant formulas than in human breast milk. Mothers seem consistently to have relatively high or relatively low concentrations of spermine and spermidine in their milk. These individual variations may be due to diet, lifestyle or genetic background; (2) the probability of developing allergy can reach $80 \%$ if the mean spermine concentration in the milk is lower than $2 \mathrm{nmol} / \mathrm{ml} \mathrm{milk}$. It is approximately $0 \%$ if the mean spermine concentration is higher than $13 \mathrm{nmol} / \mathrm{ml} \mathrm{milk;} \mathrm{(3)} \mathrm{preliminary} \mathrm{results}$ show that the intestinal permeability to macromolecules differs in premature babies when they are fed on breast milk compared with infant formulas (J Senterre, J Rigo, G Forget, G Dandrifosse and $\mathrm{N}$ Romain, unpublished results). This difference does not seem to be present when powdered milk is supplemented with polyamines at the concentration found in breast milk; (4) spermine increases proliferation and differentiation of lymphocytes isolated from the tonsils of children.
\end{abstract}

Polyamine: Milk: Allergy: Preventive medicine

Polyamines (putrescine, spermine and spermidine) can be synthesized by all eukaryotic cells (Pegg, 1986) and thus their 'essential' character (the necessity to find them in the diet) is unclear, and is the object of important studies. Nevertheless, it appears that in particular situations an exogenous supply of polyamines is necessary. For example, recently it was proposed that milk spermine or spermidine control the development of food allergy in neonates (Dandrifosse et al. 1991).

Adverse reactions represent a very wide spectrum of reaction to foods. Food intolerance is a nonpsychologically-mediated untoward reaction induced by foodstuff. Food allergy, a category of adverse reactions, is a hypersensitive state acquired through exposure to a particular allergen, re-exposure inducing an altered capacity to react through an immune response. The incidence of food intolerance depends on the method of assessment used. In response to a questionnaire the number of people who think they have experienced adverse reactions to food may be as high as $33 \%$ (Bender \& Matthews, 1981). However, when appropriate tests are used, this percentage decreases sharply. The prevalence of cow's milk allergy and intolerance has been examined in a number of retrospective studies in children, and estimates of prevalence were found to vary from 0.1 to $7.5 \%$ (Lessof, 1993). Although authors estimate that a more realistic value is lower (Department of Health, 1991), the incidence increases if other origins of food allergy are considered.

The main factors favouring the appearance of food allergy, in addition to genetic predisposition, are intestinal permeability to macromolecules and lack of maturity of the submucosal immunological system (Iynkaran \& Yadav, 1987). Indeed, in order to trigger an adverse reaction to food which involves the immune system, especially type I allergy according to the Gell \& Coombs (1963) classification, the antigenic material has to cross from the intestinal lumen to the mucosa, submucosa or the blood circulation, while retaining its allergenicity. Thus, one of the major factors in the development of allergic reactions to food components is the amount of allergens reaching the immune system. 
Passage of antigens across the intestinal epithelium would be especially easy in premature babies and in babies born at term and younger than 2 months old; the postnatal maturation of the intestine would not be complete in these children. Consequently, the likelihood of uptake of sufficient amounts of allergens to trigger allergic breakthrough is increased with an immature intestine.

When an antigen crosses the intestinal epithelium, it can be presented by macrophages to $\mathrm{T}$ lymphocytes located in the Peyer's patches. Several types of B- and T-cells would be activated. B-cells then migrate to the mesenteric lymph nodes. After further differentiation there, they return via the circulatory system to the mucosa and mature into immunoglobulin $\mathrm{A}$ or $\mathrm{E}$ antibody-producing cells. Imbalances in $\mathrm{T}$-cell regulation, as a consequence of developmental immaturity, would result in altered immune responses with associated pathology. This process would be facilitated by the relative immaturity of the baby's immunocompetent intestinal cells.

\section{Information from studies performed using sucking rats}

The rationale for examining whether polyamines have a protective role in the prevention of allergy is based on data which are directly related to the facts summarized earlier. These data have two origins, one is related to rodent studies and the second to human subjects.

In sucking rats we and other workers have demonstrated that: (1) oral administration of spermine or spermidine can induce early postnatal maturation of the intestine, i.e. in addition to modification of the specific activities of several disaccharidases (Dufour et al. 1988; Buts et al. 1993), the expression of different proteins (O Peulen and $G$ Dandrifosse, unpublished results), the level of the secretory component of s-immunoglobulin A (Buts et al. 1993), histological aspects of the intestinal epithelium (Georges et al. 1990) and intestinal permeability to macromolecules was affected by the ingestion of polyamines (Wery et al. 1992); (2) the administration of spermine at appropriate doses induced an increase in the specific activity of proteases contained in the pancreas or associated with the enterocyte brush borders (Dandrifosse et al. 1991; Wery et al. 1996a; Romain et al. 1998), suggesting that better digestion of proteins and thus of allergens could be achieved in the chyme; (3) the ingestion of spermine changes the number of cells in the cell cycle and the biochemical properties of the liver, in a manner similar to that observed at weaning (Wery et al. 1996b). For example, the synthesis of the secretory component was increased by this treatment; (4) the immunological system could be influenced by spermine ingestion (Kaouass et al. 1997b; O Peulen and $\mathrm{G}$ Dandrifosse, unpublished results). This last possibility is confirmed by studies performed in sucking mice. Ter Steege et al. (1997) have shown that after spermine ingestion there was an increase in the percentage of intra-epithelial lymphocytes expressing TCRab, CD4, CD5 and CD54, as well as the levels of expression of these antigens, which was similar to that during natural maturation.

In the rat the most recent findings in the field (for example, see Dufour et al. 1988; Georges et al. 1990; Dandrifosse et al. 1991; Romain et al. 1992, 1998; Wery et al. 1992, 1996a,b; Buts et al. 1993, 1994; Kaouass et al. 1993, 1994, 1996, 1997a,b; Deloyer et al. 1996; Peulen et al. 1997, 1998a,b; Laschet et al. 1998) have led to a working hypothesis explaining the effect of spermine on the maturation of the digestive tract, especially at the level of the intestine (Luk et al. 1980; Dufour et al. 1988; Georges et al. 1990; Buts et al. 1993; Dandrifosse et al. 1999). In this organ two phases characterize the effect of spermine (Wery et al. 1992, 1996a; Wery \& Dandrifosse, 1993; Kaouass et al. 1996; Peulen et al. 1998b; Dandrifosse et al. 1999; Peulen \& Dandrifosse, 1999); desquamation of the intestinal epithelium is followed by a hormonal cascade.

In the early phase spermine would first enter the enterocytes where it would induce apoptosis (Peulen et al. 1997, 1998a), which would also be influenced by cytokines, the secretion of which increases (Kaouass et al. 1997b; Peulen \& Dandrifosse, 1999). Second, the high permeability of the intestinal epithelium characteristic of the sucking rats would allow spermine to enter into the intercellular space, where it would react with different receptors situated on the nervous system (Kaouass et al. 1997a; Laschet et al. 1998), on the immune system (El Khefif et al. 1995; Kaouass et al. 1997b; Ter Steege et al. 1997) and on the endothelial cells, at least those at the capillary ends. The nervous system target has not been identified, but we know that spermine can be transformed into $\gamma$-aminobutyric acid (Laschet et al. 1998) and can act directly on the $\gamma$-aminobutyric acid receptor. Results also suggest that neurosecretion of bombesin is affected (Kaouass et al. 1997a). As a result, intestinal motility would be decreased, as observed in vitro in rat pups and reported in adult animals. Bile salts would remain in the lumen of the bowel, speeding up the desquamation of the tips of the villi. Alternatively, spermine and aldehydes derived from polyamines by metabolization would act on the immune system or epithelial cells, where they would induce the secretion of various cytokines such as interleukins $1 \beta$ and 6 and tumour necrosis factor $\alpha$ (Kaouass et al. 1997b; Peulen et al. 1998b). This type of secretion has been observed after spermine administration, and could cause, for example, the production of NO and the oedema noted at the tips of the villi (O Peulen and G Dandrifosse, unpublished results), a phenomenon eventually reinforced by an inhibition of ATPase activities by spermine. The latter effect would inhibit the return of exudates to the capillaries, according to the well-known Starling's (1896) law. This oedema would initiate the inflammation observed, but this phenomenon could be antagonized by spermine. In our view, although not yet conclusively demonstrated, this oedema and the initiation of inflammation are side effects of spermine, since we have shown that neither anti-interleukin $1 \beta$ and antitumour necrosis factor $\alpha$ antibodies nor cyclosporin A prevent the desquamation of the intestinal epithelium (Peulen \& Dandrifosse, 1999).

Following this first sequence of events, there is an hormonal cascade (Kaouass et al. 1993, 1994, 1997a,b; Dandrifosse et al. 1999). The more plausible hypothesis, resulting from established observations, is that spermine stimulates the secretion of bombesin from enteric nerves in synaptic clefts where bombesin would act directly on the enterocytes and/or on post-synaptic nervous receptors with, 
ultimately, activation of the pituitary-adrenal axis. In addition to bombesin, interleukin 6 (see above) and possibly other cytokines not yet identified, could also indirectly affect this axis. Since the nervous system is stimulated as well as cytokine secretions, c-fos gene is activated, then adrenocorticotropic hormone and finally corticosterone are secreted. As corticosterone increases insulin secretion, which affects the permeability of the intestinal cells to spermine (Buts et al. 1994), and as the effect of polyamine on the DNA expression is well known, it may be inferred that another pattern of enterocyte differentiation occurs which moves towards the adult stage. The increase in the intracellular spermine concentration also results from an indirect effect of corticosterone on the ornithine decarboxylase activity of the stem cells.

The molecular mechanism involved in the maturation of the pancreas and the liver after spermine ingestion remains to be explained. As this effect is not induced by intraperitoneal, intravenous or intramuscular administration of spermine (Kaouass et al. 1994, 1997a), but only by ingestion of this product, we have proposed that the hormone cascade described earlier is mainly responsible for the maturation of the digestive system.

When natural maturation occurs the same phenomena are involved (Peulen et al. 1998b). The effects are less marked and difficult to demonstrate, except the increase in spermine, the secretion of several hormones (corticosterone, tumour necrosis factor $\alpha$ and insulin), the variation in polyamine concentration in the enterocytes, the modification of gene expression, the apoptosis and the oedema (Peulen et al. 1997, 1998a; O Peulen and G Dandrifosse, unpublished results). The irreversibility of the natural process is not understood but could be associated with the biological clock, which is often cited as the basis for the intestinal maturation process.

\section{Information from studies performed with children}

All data described so far explain what occurs in the digestive tract after spermine ingestion in rat pups, but do not prove that polyamines could be preventive agents of food allergy. After birth, the maturation of the digestive system takes place (characterized by a change in gene expression in the intestine, a decrease in intestinal macromolecular permeability, and maturation of the liver and the immune system), which could be controlled by polyamine ingestion.

The following data support the suggestion that polyamines may prevent against food allergy in infancy and childhood.

\section{Polyamines and human milk}

Based on the findings reported earlier, it appears that a high polyamine content in the food given to babies could be very important in promoting the final steps in the maturation of the intestine. It is now generally accepted that the intestines of healthy term infants are more permeable to food proteins during the first 3 months than later in life (Iynkaran \& Yadav, 1987; Dandrifosse et al. 1991). It is acknowledged that during the period which follows birth antigenic macromolecules can penetrate the small intestinal mucosal membranes in quantities that may be of immunological importance. It has been noted also that allergy problems seem to be less frequent in breast-fed infants when compared with bottle-fed infants (Iynkaran \& Yadav, 1987).

This last observation suggests that when compared with breast milk infant formulas might lack one or more of the compounds important for intestinal maturation, e.g. spermine or spermidine. Thus, the polyamine concentration in infant formulas and in breast milk was estimated. The putrescine concentration was highly variable, but values for infant formulas were sometimes higher than those for human milk (Romain et al. 1992). The spermine concentration was always lower in infant formulas than in breast milk. The spermidine concentration of breast milk was also higher than that of infant formulas. These results agree with observations reported by other authors (Pollack et al. 1992; Buts et al. 1995), and indicate that the spermine and spermidine intake of breast-fed babies is higher than that of formula-fed babies. These findings have been confirmed by Dorhout et al. (1996).

From these results it may be proposed that the possible protective effect of breast milk against allergies could be explained, at least partially, by its high level of polyamines, especially spermine.

\section{Epidemiological analysis}

A large individual variation in the polyamine concentration of breast milk has been recorded, even though values were obtained under the same conditions (milk samples were taken from the second breast just before the baby began sucking; Romain et al. 1992). The concentrations of putrescine, spermine and spermidine in breast milk seemed to be consistently either relatively high or relatively low. These individual variations may be due, for example, to the diet, the lifestyle or the genetic background of each mother. In rat dams, on the other hand, we found that the spermine concentration of the milk is dependent on the composition of the diet (O Peulen and G Dandrifosse, unpublished results), i.e. the concentration is influenced by the spermidine, arginine and methionine content of the food.

The possible implications of polyamines in breast milk for the health of babies, especially as agents against allergy, have been evaluated (Peulen et al. 1998c). At 5 years after the birth of their child a questionnaire was sent to mothers who breast-fed and gave samples of their milk for the measurement of polyamine concentration (see Romain et al. 1992). Forty-five mothers responded. By this method information was obtained about the duration of lactation and the eventual outcome in relation to food or environmental allergy in the child. Results were confirmed or invalidated by the mother's general practitioner who was free to choose the method of diagnosis. The diagnosis was totally blind to the polyamine status of the child.

There appeared to be no difference between responders and non-responders with respect to allergy or the polyamine content of their milk. In the responders $29 \%$ had an allergic child. Although this percentage is high relative to that in the general population, indicating that the sample is therefore not representative, the conclusions are still valid since they 
were based on the presence or absence of allergy in each child.

The results indicate that children with an allergy when they were 5 years old consumed a milk which contained less spermine and spermidine than that of the children who showed no allergy at the same age, a fact which can be explained by a better postnatal maturation of the small intestine and the immune system; the greater maturity of the small intestine leads to gut closure, which decreases the transfer of antigens from the lumen to the blood (see p. 81).

A mathematical model describing the situation was proposed. The model suggests that the development of allergy is dependent on the mean spermine concentration in the milk consumed during the first postnatal month. The sensitivity of this model is high ( $88 \%$, true negative) but the specificity is lower ( $54 \%$, true positive), indicating that the model is better at identifying non-allergic children than allergic children. This observation is probably due to the well-known multifactorial origin of allergy, which includes the low spermine concentration of milk. As reported earlier, the spermine in the milk will only be a preventive agent when acting as controller of the maturation of the small intestine.

The critical value above which children have a reduced risk of allergy is $5.02 \mathrm{nmol}$ spermine/ $/ \mathrm{ml}$. If the children were classed as allergic or not allergic according to the mean spermine concentration in the milk they consumed, the specificity of the model reaches $69 \%$ (true positive) and sensitivity $92 \%$ (true negative). This result indicates that $5.02 \mathrm{nmol}$ spermine $/ \mathrm{ml}$ could be used as an early diagnosis tool to determine whether a child will develop allergy later. Thus, $69 \%$ of the children who will become allergic could be detected early and treated with an hypoallergenic diet. Furthermore, as spermine is not toxic at physiological doses, it could be added to formulas to prevent the development of the disease.

In conclusion, these studies show that allergy could be prevented in children by modifying the composition of their mother's milk. Moreover, children who are susceptible to allergy could be detected very early by an analysis of their mother's milk. Their diet or environment could be modified to reduce the risk of developing the condition. Nevertheless further studies are clearly necessary.

\section{Polyamines and the intestinal immune system in human subjects}

It has been shown that the number of $\mathrm{T}$ helper cells (CD4): the number of $\mathrm{T}$ suppresser cells is about $8: 1$ in newborn infants and about $3: 1$ in adults, and it has been suggested that some children are excessively sensitized to food antigens because of a relative deficiency of suppresser mechanisms and/or because of an imbalance in immune regulatory systems. Impaired regulation of the relative amounts of immune cells could induce an inadequate response of these systems and the pathology associated with them. Polyamines could play a role in these phenomena.

The effect of spermine and/or spermidine on lymphocyte differentiation and proliferation in the presence or absence of a food allergen has been studied (El Khefif et al. 1995;
N El Khefif and G Dandrifosse, unpublished results). Lymphocytes were isolated from the tonsils of atopic or non-atopic children who were less than 8 years old. The effect of spermine was tested on these cells which were maintained in primary cultures. The cells were cultivated in presence of mitogens (phytohaemagglutinin $\mathrm{L}$ and $\mathrm{M}$, phorhol myristate acetate, concanavalin A and lipopolysaccharide) and/or allergens. They were cultivated for $24 \mathrm{~h}$ before receiving $\left[{ }^{3} \mathrm{H}\right]$ thymidine. They were then maintained alive for $16 \mathrm{~h}$ before being analysed.

Spermine $(0 \cdot 1-10 \mathrm{mM})$ inhibited DNA synthesis by lymphocytes stimulated by phytohaemagglutinin L, but only in presence of bovine serum ( $\mathrm{N}$ El Khefif and G Dandrifosse, unpublished results). This effect seems to be due to spermine itself and not to aldehyde derivatives of this polyamine, as MDL 72.527 (N1, N4-bis (2,3-butanedienyl) 1,4-butanediamine, an inhibitor of polyamine oxidase activity) did not inhibit the effect of spermine on the DNA synthesis. In a serum-free medium which supports in vitro proliferation of human T-cells stimulated by the same mitogen, spermine induced proliferation of these cells. Spermine also acted on the differentiation of the T-cells. Indeed, when spermine was added to the culture media a decrease in the expression of the interleukin 2 receptor $\left(\mathrm{CD}_{25}\right.$ marker) and the transferrin receptor $\left(\mathrm{CD}_{71}\right.$ marker) was observed, but $\mathrm{CD}_{4}: \mathrm{CD}_{8}$ was unchanged. These results indicate that the proliferation of $\mathrm{T}$ suppresser lymphocytes is not modified by spermine under our experimental conditions. The modification of lymphocyte activity is more probably due to a difference in expression and/or in translation of the genes for the receptors concerned $\left(\mathrm{CD}_{25}\right.$ and $C D_{71}$ ). It remains to be determined whether the process differs depending on the origin of the lymphocytes (atopic or non-atopic children) and the experimental conditions chosen.

In the presence of Staphylococcus aureus Cowen strain I the proliferation of B lymphocytes was stimulated, but the expression of the $\mathrm{CD}_{23}$ marker for these cells was not affected by spermine.

Although preliminary, these observations present arguments which support a role for spermine in the maturation of the gut-associated lymphoid tissue system in children, and subsequently in the development of food allergy.

\section{Intestinal permeability to macromolecules}

It is well-known that intestinal permeability to allergens (generally macromolecules) varies after birth in children (Axelsson et al. 1989). Since it has been shown in the sucking rats that intestinal permeability is modulated by spermine ingestion (see pp. 82-83), it appears reasonable to suggest that this polyamine contained in milk could have the same role in infants. Thus, we have tried to find a suitable marker for intestinal permeability. In preliminary experiments the intestinal permeability to macromolecules in healthy children and in children with different diseases was estimated using human $\alpha$-lactalbumin which was presented in a specific natural form.

Of the children studied, $26 \%$ ( $n$ 23) had human $\alpha$-lactalbumin in their serum before ingestion of this 
protein. This finding could be explained by the ingestion of breast milk before the test, but only one child had consumed breast milk before visiting the clinic. Another explanation could be that the rabbit anti-human $\alpha$-lactalbumin antibody used was contained in a total serum. Consequently, other antibodies could recognize proteins other than human $\alpha$-lactalbumin in the human serum. The results were adjusted accordingly. The observations showed that the children without disease $(n 4)$ and $86 \%$ $(n 7)$ of the children with a non-allergy disease had no intestinal permeability to macromolecules. Only one child with diarrhoea had a high intestinal permeability to human $\alpha$-lactalbumin. Of the children with an allergy and not deprived of the allergens to which they were sensitive, $75 \%$ (n 4) had a high intestinal permeability to macromolecules. Of the children with an allergic disease but deprived of allergens to which they were sensitive, $60 \%(n 5)$ had an intestinal permeability which was zero. However, $20 \%$ ( $n$ 5) of the allergic children deprived of allergens had a low, but not zero, intestinal permeability to human $\alpha$-lactalbumin. Of the children suspected of allergy, $33 \%$ ( $n$ 3) had a high intestinal permeability to macromolecules, $33 \%$ had a low permeability and $33 \%$ had a permeability which was zero.

If we do not consider the results relating to children only suspected of allergy, $74 \%$ of results are in agreement with the expected results. The technique used reveals that intestinal permeability to macromolecules is increased in children with an allergic disease, as reported by other authors (Juvonen et al. 1990; Andre et al. 1991). The technique also shows that food intolerance does not seem to induce a high intestinal permeability to macromolecules. However, other authors (Eaton et al. 1995) have reported that intestinal permeability is increased in patients with this type of disease. The difference in the results between the two studies can be easily explained, since Eaton et al. (1995) measured intestinal permeability using polyethylene glycol, i.e. the over-absorption was only significant with substances having a low molecular weight. In mucoviscidosis the intestinal permeability to human $\alpha$-lactalbumin does not seem to be increased, a result which could be explained by the excess mucus produced.

A similar approach was used in premature babies (G Forget, J Rigo, G Dandrifosse, N Romain and O Peulen, unpublished results). Babies receiving infant formulas either non-enriched or enriched with spermine (or/and spermidine) were studied for intestinal permeability to human $\alpha$-lactalbumin. Preliminary results indicate that the intestinal permeability to macromolecules could be different in premature babies depending on whether they receive maternal breast milk or powdered milk with or without added spermine.

In conclusion, in view of the observations described in the present paper for animals and human subjects, it would seem reasonable to suggest that insufficient polyamine intake could play a role in the induction of sensitization to dietary allergens in infancy and childhood. Nevertheless, additional research is needed to confirm whether dietary supplementation with polyamines would reduce the incidence of food allergy in the infant.

\section{Acknowledgements}

MDL 72.527 was a generous gift from Dr Seiler (Merrel Dow Research Institute, Strasbourg, France).

\section{References}

Andre F, Andre C, Feknous M, Colin L \& Cavagna S (1991) Digestive permeability to different-sized molecules and to sodium cromoglycate in food allergy. Allergy Proceedings 12, 293-298.

Axelsson I, Jakobsson I, Lindberg T, Polberger S, Benediktsson B \& Räihä N (1989) Macromolecular absorption in preterm and term infants. Acta Paediatrica Scandinavica 78, 532-537.

Bender AE \& Matthews DR (1981) Adverse reactions to foods. British Journal of Nutrition 46, 403-407.

Buts JP, De Keyser N, De Raedemaeker L, Collette E \& Sokal EM (1995) Polyamine profiles in human milk, infant formulas, and semi-elemental diets. Journal of Pediatric Gastroenterology and Nutrition 21, 44-49.

Buts JP, De Keyser N, Kolanowski J, Sokal E \& Van Hoof F (1993) Maturation of villus and crypt cell functions in rat small intestine. Role of dietary polyamines. Digestive Diseases and Sciences 38, 1091-1098.

Buts J-P, De Keyser N, Romain N, Dandrifosse G, Sokal E \& Nsengiyumva T (1994) Response of rat immature enterocytes to insulin: regulation by receptor binding and uptake of endoluminal polyamine uptake. Gastroenterology 106, 49-59.

Coombes RRA \& Gell PGH (1963) The classification of allergic reactions underlying disease. In Clinical Aspects of Immunology, pp. 313-317 [PGH Gell and RRA Coombes, editors]. Oxford: Blackwell Science.

Dandrifosse G, Deloyer P, El Khefif N \& Peulen O (1999) Dietary polyamines during lactation. In Polyamines in Health and Nutrition, pp. 213-231 [S Bardocz and A White, editors]. Boston: Kluwer Academic Publishers.

Dandrifosse G, Forget G, Romain N, Deloyer P \& Wery I (1991) Maturation précoce de l'intestin du rat par la spermine: résultats et perspectives (Early maturation of rat intestine by spermine: results and prospects). In Les Polyamines: Chimie, Biologie, Médecine (Polyamines: Chemistry, Biology, Medicine), pp. 237-247 [JP Moulinoux and V Quemener, editors]. Paris: Flammarion.

Deloyer P, Dandrifosse G, Bartholomeus C, Romain N, Klimek M, Salmon J, Gérard P, Goessens G \& Eyssen H (1996) Polyamine and intestinal properties in adult rats. British Journal of Nutrition 76, 627-637.

Department of Health (1991) ACNFP Guidelines on the Assessment of Novel Foods and Processes. Report on Health and Social Subjects no. 38. London: HM Stationery Office.

Dorhout B, van Beusekom M, Huisman M, Kingma AW, de Hoog E, Boersma ER \& Muskiet FAJ (1996) Estimation of twenty-four hour polyamine intake from mature human milk. Journal of Pediatric Gastroenterology and Nutrition 23, 298-302.

Dufour C, Dandrifosse G, Forget P, Vermesse F, Romain N \& Lepoint P (1988) Spermine and spermidine induce intestinal maturation in the rat. Gastroenterology 95, 112-116.

Eaton KK, Howard M \& McLaren-Howard JMH (1995) Gut permeability measured by polyethylene glycol absorption in abnormal gut fermentation as compared with food intolerance. Journal of the Royal Society of Medicine 88, 63-66.

El Khefif N, Peulen O \& Dandrifosse G (1995) La spermine induit la prolifération de lymphocytes humains (Spermine induces the proliferation of human lymphocytes). Archives of Physiology and Biochemistry 103, D116.

Georges P, Dandrifosse G, Vermesse F, Forget P, Deloyer P \& Romain N (1990) Reversibility of spermine-induced intestinal 
maturation in the rat. Digestive Diseases and Sciences $\mathbf{3 5}$, $1528-1536$

Iynkaran N \& Yadav M (1987) Food allergy. In Food Allergy. Immunopathology of the Small Intestine, pp. 415-449 [MN Marsch, editor]. New York: John Wiley and Sons Ltd.

Juvonen P, Jakobsson I \& Lindberg T (1990) Macromolecular absorption and cows' milk allergy. Archives of Disease in Childhood 65, 300-303.

Kaouass M, Deloyer P \& Dandrifosse G (1993) Intestinal development in suckling rats: direct or indirect spermine action? Digestion 55, 160-167.

Kaouass M, Deloyer P \& Dandrifosse G (1997a) Involvement of bombesin in spermine-induced corticosterone secretion and intestinal maturation in suckling rats. Journal of Endocrinology 153, 429-436.

Kaouass M, Deloyer P, Gouders I, Peulen O \& Dandrifosse G (1997b) Role of interleukin-1 beta, interleukin-6, and TNF alpha in intestinal maturation induced by dietary spermine in rats. Endocrine 6, 187-194.

Kaouass M, Deloyer P, Wery I \& Dandrifosse G (1996) Analysis of structural and biochemical events occurring in the small intestine after dietary polyamine ingestion in suckling rats. Digestive Diseases and Sciences 41, 1434-1444.

Kaouass M, Sulon J, Deloyer P \& Dandrifosse G (1994) Spermine-induced precocious intestinal maturation in suckling rats: possible involvement of glucocorticoids. Journal of Endocrinology 141, 279-283.

Laschet J, El Khefif N, Dandrifosse G, Evrard B, Minier F, Moulinoux JP, Seiler N \& Bureau M (1998) Spermine and other polyamines as intracellular modulators of the endogenous phosphorylation of $\mathrm{GABA}_{\mathrm{A}}$ receptors. In Biologically Active Amines in Food. vol. 2: Metabolic Effects of Biologically Active Amines, pp. 86-94 [S Bardocz, A White and G Hajos, editors]. Luxembourg: European Commission Directorate-General XII Science, Research and Development.

Lessof M (1993) Food Allergy and Other Adverse Reactions to Food. Brussels: ILSI Europe Publications.

Luk GD, Marton LJ \& Baylin SB (1980) Ornithine decarboxylase is important in intestinal mucosal maturation and recovery from injury in rats. Science 210, 195-198.

Pegg AE (1986) Recent advances in the biochemistry of polyamines in eukaryotes. Biochemical Journal 234, 249-262.

Peulen O \& Dandrifosse G (1999) Cyclosporin A inhibits spermine induced differentiation but not cell loss of suckling rat small intestine. Digestive Diseases and Sciences (In the Press).
Peulen O, Dewe W, Dandrifosse G, Henrottay I \& Romain N (1998c) The relationship between spermine content of human milk during the first postnatal month and allergy in children. Public Health Nutrition 1, 184-188.

Peulen O, Muller P, Defresne M-P \& Dandrifosse G (1997) Spermine induced apoptosis in suckling rat small intestinal mucosa. Archives of Physiology and Biochemistry 105, 252-253.

Peulen O, Muller P, Gérard P \& Dandrifosse G (1998a) Spermineinduced apoptosis in the small intestine of suckling rats. Archives of Physiology and Biochemistry 106, 1-29.

Peulen O, Pirlet C, Klimek M \& Dandrifosse G (1998b) Comparison between the natural postnatal maturation and the spermine-induced maturation of the rat intestine. Archives of Physiology and Biochemistry 106, 46-55.

Pollack PF, Koldovsky O \& Nishioka K (1992) Polyamines in human and rat milk and in infant formulas. American Journal of Clinical Nutrition 56, 371-375.

Romain N, Dandrifosse G, Jeusette F \& Forget P (1992) Polyamine concentration in rat milk and food, human milk and infant formulae. Pediatric Research 32, 58-63.

Romain N, Gesell MS, Leroy O, Forget P, Dandrifosse G \& Luk GD (1998) Effect of spermine administration on pancreatic maturation in unweaned rats. Comparative Biochemistry and Physiology 120A, 379-384.

Starling EH (1896) On the absorption of fluids from the connective tissue space. Journal of Physiology 19, 312-326.

Ter Steege JC, Buurman WA \& Forget PP (1997) Spermine induces maturation of the immature intestinal immune system in neonatal mice. Journal of Pediatric Gastroenterology and Nutrition 25, $332-340$.

Wery I \& Dandrifosse G (1993) Evolution of biochemical parameters characterizing the proximal small intestine after orally administered spermine in unweaned rats. Endocrine Regulations 27, 201-207.

Wery I, Deloyer P \& Dandrifosse G (1996a) Effect of a single dose of orally-administered spermine on the intestinal development of unweaned rats. Archives Internationales de Physiologie et de Biochimie 104, 163-172.

Wery I, Kaouass M, Deloyer P, Buts JP, Barbason H \& Dandrifosse $\mathrm{G}$ (1996b) Exogenous spermine induces maturation of the liver in suckling rats. Hepatology 24, 1206-1210.

Wery I, Romain N \& Dandrifosse G (1992) Maturation intestinale précoce induite par la spermine chez le rat: cinétique et perméabilité intestinale (Early intestinal maturation induced by spermine in the rat: intestinal kinetics and permeability). Archives Internationales de Physiologie et de Biochimie 101, A135. 